kann insofern nichts anderes gelten. Verbietet das für den Verweisungsvertrag danach maßgebliche Statut (wie etwa das deutsche Recht in Allgemeinen Geschäftsbedingungen) eine geltungserhaltende Reduktion einer überschießenden Klausel, droht damit ihre vollständige Unwirksamkeit, falls nach Streichung des unwirksamen Bestandteils keine sinnvolle Restklausel verbleibt. ${ }^{32}$

\section{Klauselvorschläge}

Die nachstehenden Klauselvorschläge setzen die dargestellten Rahmenbedingungen des Europäischen Kollisionsrechts bei der Gestaltung von Rechtswahlklauseln im unternehmerischen Geschäftsverkehr um. Angesichts der Risiken einer Gesamtunwirksamkeit wird im Absatz 1 der vorgeschlagenen Musterklausel ausschließlich das Vertragsstatut gewählt und bewusst auf eine weitergehende Formulierung, nach der beispielsweise „alle aus oder im Zusammenhang mit dem Vertrag“ entstehenden Rechtsfragen der Rechtswahl unterfallen sollen, verzichtet. Sofern der Anwendungsbereich des UN-Kaufrechts (CISG) eröffnet ist, empfiehlt sich wie im Klauselvorschlag vorgesehen zudem eine klare Festlegung, ob das CISG (wie gem. Art. 6 CISG zulässig) ausgeschlossen werden soll oder nicht. ${ }^{33}$ Die in der Rom I-VO vorgesehene Sonderanknüpfung an das Recht des Erfüllungsortes gem. Art. 12 Abs. 2 Rom I-VO wird aus den dargestellten Gründen ausdrücklich ausgeschlossen.

Im zweiten Absatz der Musterklausel wird das im ersten Absatz gewählte Recht auch auf konkurrierende außervertragliche Ansprüche erstreckt. Soweit eine Rechtswahl in AGB überhaupt nicht zulässig sein sollte, bleibt der erste Absatz der Rechtswahlklausel auch bei Anwendung eines blue pencil Tests grundsätzlich unversehrt. Zur weiteren Absicherung gegen eine Unwirksamkeit werden allerdings diejenigen Rechtsbereiche, die einer
Rechtswahl kollisionsrechtlich nicht zugänglich sind, ausdrücklich vom Geltungsbereich der Klausel ausgenommen.

$\$[\bullet]$ Anwendbares Recht

(1) Dieser Vertrag unterliegt dem Recht von [•][ggf. Zusatz im Fall internationaler Lieferverträge: unter Einschluss/ Ausschluss des UN-Kaufrechts (CISG)]. Die Anwendung von Art. 12 Abs. 2 Rom I Verordnung ist ausgeschlossen.

(2) Das nach Absatz 1 gewählte Recht ist mit Ausnahme von Ansprüchen, die auf unlauterem Wettbewerbsverhalten, aus einem den freien Wettbewerb einschränkenden Verhalten oder einer Verletzung von Rechten des geistigen Eigentums beruhen, auch auf außervertragliche Schuldverhältnisse anwendbar, die aus oder im Zusammenhang mit der Durchführung dieses Vertrages entstehen.

$\S[\bullet]$ Governing Law

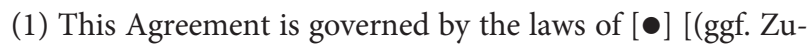
satz im Fall internationaler Lieferverträge: to the inclusion/to the exclusion of the United Nations Convention on Contracts for the International Sales of Goods (CISG)]. The application of Art. 12 (2) Rome I Regulation is excluded.

(2) This choice of law applies (to the exclusion of claims based on an act of unfair competition, the restriction of competition or an infringement of an intellectual property right pursuant to Arts. 6 (4) and 8 (3) Rome II Regulation) also with regard to any concurring claims under tort arising out of or related to the performance of this Agreement.

32 So für die Wirkung des Verbotes der geltungserhaltenden Reduktion im deutschen Recht etwa BGH, NJW 1989, 3215.

33 Die Prüfung der Anwendbarkeit der CISG erfolgt grs. vorrangig vor dem Kollisionsrecht, was entweder aus Art. 25 Rom I-VO oder aber bereits aus dem Umstand abgeleitet werden kann, dass es in diesem Fall an einer Kollision mehrerer Sachrechtsordnungen mangelt, s. dazu Schilling, EuZW 2011, 776, 778

\title{
Die Geltungsbereiche des UN-Kaufrechts und des Gemeinsamen Europäischen Kaufrechts im Vergleich
}

\author{
Professor Dr. Phillip Hellwege, Augsburg*
}

\section{Einleitung}

Das UN-Kaufrecht ist in fast allen Mitgliedstaaten der Europäischen Union sowie in allen Staaten, die zu ihren Beitrittskandidaten zählen, in Kraft getreten, zuletzt am 1.8.2011 in der Türkei. Außen vor bleiben weiterhin das Vereinigte Königreich, Irland, Malta und Portugal. ${ }^{1}$ Das UN-Kaufrecht bietet damit für einen Großteil Europas und darüber hinaus Regeln für grenzüberschreitende Kaufverträge an. Doch könnte es schon bald Konkurrenz bekommen. Am 11.10.2011 legte die Europäische Kommission einen Vorschlag für eine Verordnung über ein Gemeinsames Europäisches Kaufrecht ${ }^{2}$ vor. ${ }^{3}$ Auch das Gemeinsame Europäische Kaufrecht soll Regeln für grenzüberschreitende Kaufverträge
* Der Beitrag wird im nächsten Heft der IHR unter dem Titel „UN-Kaufrecht oder Gemeinsames Europäisches Kaufrecht?“ fortgesetzt. Beide Beiträge zusammen basieren auf einem Vortrag, den der Autor am 12.4.2012 beim gemeinsam von den juristischen Fakultäten der Universitäten Ankara und Augsburg veranstalteten 2. Deutsch-Türkischen Rechtssymposium in Ankara gehalten hat. Das Manuskript wurde am 11.4.2012 abgeschlossen. Danach erschienene Literatur wurde für diese veröffentlichte Version noch berücksichtigt, sofern sie den Autor bis zum 11.5.2012 erreicht hat.

1 Zu den Gründen ihres Nichtbeitritts vgl. Schroeter, UN-Kaufrecht und Europäisches Gemeinschaftsrecht, 2005, S. 21 ff. Zur Position in England vgl. Illmer, in: Triebel/Illmer/Ringe/Vogenauer/Ziegler (Hrsg.), Englisches Handels und Wirtschaftsrecht, 3. Aufl. 2012, S. 91.

2 Vorschlag für eine Verordnung des Europäischen Parlaments und des Rates über ein Gemeinsames Europäisches Kaufrecht, 11.10.2011, KOM (2011) 635 endg. Abgedruckt in: Schulze/Zimmermann (Hrsg.), Europäisches Privatrecht. Basistexte, 4. Aufl. 2012, S. 43-105. 
in Europa zur Verfügung stellen. An der Umsetzung des Vorhabens eines Gemeinsamen Europäischen Kaufrechts bestehen kaum mehr Zweifel. ${ }^{4}$ Nach Inkrafttreten eines Gemeinsamen Europäischen Kaufrechts wird sich den Marktteilnehmern in Europa die Frage stellen, ob sie dieses, das UN-Kaufrecht oder allein ein nationales Recht zur Anwendung kommen lassen wollen. ${ }^{5}$ Freilich stellt sich den Marktteilnehmer diese Frage nur in den Fällen, in denen sowohl das UN-Kaufrecht als auch das Gemeinsame Europäische Kaufrecht an- bzw. verwendbar sind. Ziel dieses Beitrages ist es, diese Schnittmengen herauszuarbeiten.

Die Vorschriften zum Anwendungsbereich des UN-Kaufrechts finden sich in den Artt. 1-7 CISG. Der Verordnungsvorschlag zerfällt in zwei Teile. Der Entwurf des Gemeinsamen Europäischen Kaufrechts (im Weiteren: DCESL für Draft Common European Sales Law) ist im Anhang des Verordnungsvorschlages verortet. Der Verordnungsvorschlag selbst (im Weiteren: DCESL-VO) enthält vor allem Definitionen und eben Bestimmungen über die mögliche Verwendbarkeit des Gemeinsamen Europäischen Kaufrechts.

\section{Niederlassung, gewöhnlicher Aufenthalt, Anschriften}

Nach UN-Kaufrecht und Gemeinsamem Europäischen Kaufrecht ist gleichermaßen die Staatsangehörigkeit der Vertragsparteien ohne Bedeutung. Für das UN-Kaufrecht stellt dies Art. 1(3) CISG explizit fest. ${ }^{6}$ Entscheidend ist nach Art. 1(1) CISG allein, dass die Vertragsparteien ihre Niederlassung in verschiedenen Staaten haben. Der Begriff der Niederlassung deutet darauf hin, dass das UN-Kaufrecht seinen Hauptanwendungsbereich im unternehmerischen Verkehr hat. ${ }^{7}$ In diese Richtung gehen auch die nach der Unterzeichnungsklausel autoritativen Sprachfassungen. ${ }^{8}$ In der englischen Fassung heißt es etwa places of business. Fehlt es an einer Niederlassung kommt es nach Art. 10(b) CISG auf den gewöhnlichen Aufenthalt an.

Eine mit Art. 1(3) CISG vergleichbar Klarstellung enthält der Verordnungsvorschlag nicht, doch ist auch für diesen die Staatsangehörigkeit ohne Bedeutung. Nach Art. 4(1) DCESL-VO kann „das Gemeinsame Europäische Kaufrecht [...] für grenzübergreifende Verträge verwendet werden“, und Art. 4(2)-(3) DCESL-VO definiert den Begriff des grenzübergreifenden Vertrages: Bei Verträgen zwischen Unternehmern kommt es nach Art. 4(2) DCESL-VO auf ihren gewöhnlichen Aufenthalt an, der in verschiedenen Staaten liegen muss. Den Begriff des gewöhnlichen Aufenthalts bestimmt Art. 4(4)-(5) DCESL-VO näher. So kommt es nach Art. 4(4) DCESL-VO für Gesellschaften, Vereine und juristische Personen auf den Sitz der Hauptverwaltung, für natürliche Personen auf den Hauptgeschäftssitz an, und Art. 4(5) DCESL-VO enthält eine Vorschrift zu Zweigniederlassungen. Ist an dem Vertrag ein Verbraucher beteiligt, so kommt es für diesen nach Art. 4(3) DCESL-VO auf dessen Anschrift, Lieferanschrift oder Rechnungsanschrift an. Nach Art. 4(6) DCESL-VO ist schließlich für die Einordnung eines Vertrags als grenzübergreifend auf den Zeitpunkt abzustellen, „zu dem die Verwendung des Gemeinsamen Europäischen Kaufrechts“ vereinbart wurde.

Art. 4 DCESL-VO könnte aus mehreren Gründen als wenig gelungen kritisiert werden: So fällt die im Vergleich zum UN-
Kaufrecht größere Regelungsdichte auf, ohne dass damit etwas gewonnen zu sein scheint. Es findet sich eine begriffliche Vielfalt - gewöhnlicher Aufenthalt, Anschrift, Lieferanschrift, Rechnungsanschrift, Ort der Hauptverwaltung, Hauptgeschäftssitz, Niederlassung -, die vermeidbar gewesen wäre. So kommt dem Begriff des gewöhnlichen Aufenthalts prima facie keine eigenständige Bedeutung zu, wird er doch für die verschiedenen Situationen in Art. 4(4)-(5) DCESL-VO ausgefüllt. Nicht gelungen sind auch die Definitionen grenzübergreifender Verträge in Art. 4(2)-(3) DCESL-VO. Art. 4(2) DCESL-VO lautet etwa:

„Für die Zwecke dieser Verordnung ist ein Vertrag zwischen Unternehmern ein grenzübergreifender Vertrag, wenn die Parteien ihren gewöhnlichen Aufenthalt in verschiedenen Staaten haben, von denen mindestens einer ein EU-Mitgliedstaat ist."

Dass man für eine Definition eines grenzübergreifenden Vertrages daran anknüpft, dass die Parteien ihren gewöhnlichen Aufenthalt in verschiedenen Staaten haben, ist eingängig, nicht aber dass es Teil der Definition des Begriffes „grenzübergreifend“ ist, dass einer dieser Staaten ein EU-Mitgliedstaat ist. Hat eine Partei ihren gewöhnlichen Aufenthalt in China, die andere in den U.S. A., so handelt es sich ohne jeden Zweifel um einen grenzübergreifenden Vertrag, eben nur nicht um einen solchen, für den das Gemeinsame Europäische Kaufrecht verwendet werden kann. In die Definition des Begriffes ,grenzübergreifend“ gehört diese Einschränkung daher auch nicht hinein. Die Einschränkung im letzten Halbsatz des Art. 4(2) hätte in Art. 4(1) DCESL-VO aufgenommen werden sollen, obwohl dies sprachlich sicherlich Probleme aufgeworfen hätte. Aber auch wenn die Definitionen grenzübergreifender Verträge misslungen sind, wird dies nicht zu Anwendungsproblemen führen, und daher sollen diese Probleme hier auch nicht weiter vertieft werden.

Praktisch relevant ist aber, dass in Verträgen zwischen einem Verbraucher und einem Unternehmer nach Art. 4(3) DCESL-VO alternativ an die Anschrift, Liefer- oder Rechnungsanschrift angeknüpft werden kann:

„Für die Zwecke dieser Verordnung ist ein Vertrag zwischen einem Unternehmer und einem Verbraucher ein grenzübergreifender Vertrag, wenn (a) sich die Anschrift des Verbrauchers, die Lieferanschrift oder die Rechnungsanschrift in einem anderen Staat als dem Staat befindet, in dem der Unternehmer seinen gewöhnlichen Aufenthalt hat, und (b) mindestens einer dieser Staaten ein EU-Mitgliedstaat ist.“

$3 \mathrm{Zu}$ den Vorarbeiten vgl. etwa Remien, in: Remien/Herrler/Limmer (Hrsg.), Gemeinsames Europäisches Kaufrecht für die EU?, 2012, S. 1 f.; Zimmermann, JBl 2012, 4 ff.; Schulte-Nölke, in: Schulte-Nölke/Zoll/ Jansen/Schulze (Hrsg.), Der Entwurf für ein optionales europäisches Kaufrecht, 2012, S. 1 ff.; Staudenmayer, NJW 2011, 3493 ff. Einleitend zum Entwurf Schmidt-Kessel, in: ders. (Hrsg.), Ein einheitliches europäisches Kaufrecht?, 2012, S. 1 ff.

4 Vgl. nur Reding, in: Schulze/Stuyck (Hrsg.), Towards a European Contract Law, 2011, S. $18 \mathrm{ff}$.

Vgl. bereits Lando, in: Remien/Herrler/Limmer (o. Fn. 3), S. 15 ff.

6 Hierzu Ferrari, in: Schlechtriem/Schwenzer, 5. Aufl. 2008, Art.1 Rn. 59-60.

Vgl. Staudinger/Magnus, CISG, 2005, Art. 1 Rn. 125.

8 Hierzu Staudinger/Magnus (o. Fn. 7), Unterzeichnungsklausel Rn. 1. 
Die alternative Anknüpfung an die Anschrift, die Lieferanschrift oder die Rechnungsanschrift wirft zunächst die Frage auf, ob die Begriffe „Lieferanschrift“ und „Rechnungsanschrift“ die Wendung „Anschrift des Verbrauchers“ konkretisieren sollen, so dass die Vorschrift nur zwei Anschriften als maßgeblich erachtet. Doch dann wäre der Satzteil „die Lieferanschrift oder die Rechnungsanschrift" eine Apposition, die in Kommata eingeschlossen sein müsste. So handelt es sich um die Aufzählung von drei unterschiedlichen Anschriften. Für eine Aufzählung sprechen auch die anderen sprachlichen Fassungen des Verordnungsvorschlages. So spricht etwa die englische Version von „either the address indicated by the consumer, the delivery address for goods or the billing address“. Würde es sich um eine Apposition handeln, so hätte es heißen müssen: „the address indicated by the consumer, either the delivery address for goods or the billing address".

An die Stelle der subsidiären Anknüpfung an den gewöhnlichen Aufenthalt im UN-Kaufrecht tritt im Verordnungsvorschlag also für Verbraucher die alternative Anknüpfung an drei Anschriften. Damit wird wohl bezweckt, dass für den Unternehmer erkennbar sein soll, ob das Gemeinsame Europäische Kaufrecht verwendbar ist. ${ }^{9}$ Denn der gewöhnliche Aufenthalt des Verbrauchers muss ihm nicht immer hinreichend deutlich sein. Dass der Verordnungsvorschlag Rechtsklarheit herstellen will, wird freilich in den anderen Sprachfassungen sehr viel deutlicher, sprechen sie doch davon, dass die erste Anschrift vom Verbraucher angegeben worden sein muss. So heißt es in der englischen Version: „the address indicated by the consumer". Nach der deutschen Fassung scheint dagegen irgendeine Anschrift auszureichen. ${ }^{10}$ Daran ändert auch der bestimmte Artikel nichts.

Freilich führt die alternative Anknüpfung an drei unterschiedliche Adressen auch zu einer Ausdehnung der Verwendbarkeit des Kaufrechts, die zumindest dann kritisch hinterfragt werden muss, wenn man nicht ohnehin eine unbeschränkte Wahlmöglichkeit des Gemeinsamen Europäischen Kaufrechts befürwortet: ${ }^{11}$ Hält sich der Verbraucher nur vorübergehend im gleichen Staat wie der Unternehmer auf, etwa weil er dort Urlaub macht, und lässt er sich eine Kaufsache in sein Hotel liefern, wohin auch die Rechnung versandt wird, so würde es für die Verwendbarkeit des Gemeinsamen Kaufrechts schon ausreichen, wenn der Verbraucher noch zusätzlich seine Heimatanschrift angibt. Auch reicht es nach dem Verordnungsvorschlag für die Verwendbarkeit des Gemeinsamen Europäischen Kaufrechts aus, wenn der Verbraucher zwar seine Anschrift im gleichen Staat wie der Unternehmer hat und sich an diese auch beliefern lässt, als Rechnungsanschrift aber eine ausländische Adresse, etwa die eines Verwandten, angibt. ${ }^{12}$

Doch interessieren die den Art. 4 DCESL-VO umgebenden Probleme hier nicht weiter. In diesem Beitrag soll allein die Frage beantwortet werden, inwieweit sich die Anwendungsbereiche des UN-Kaufrechts und des Gemeinsamen Europäischen Kaufrechts decken. Diese decken sich zwar nicht in jedem, aber doch in einer Vielzahl von Fällen: Zwar knüpft das UN-Kaufrecht an den Begriff der Niederlassung an, der Verordnungsvorschlag dagegen für Unternehmer an den des gewöhnlichen Aufenthalts. Doch stimmen die Konkretisierungen des gewöhnlichen Aufenthalts in Art. 4(4)-(5) DCESL-VO mit der Auslegung des Niederlassungsbegriffs der Artt. 1 und 10 CISG weitgehend überein. ${ }^{13}$ Mit Blick auf Verträge zwischen Unternehmern und Verbrau- chern kommt es für das UN-Kaufrecht mangels einer Niederlassung des Verbrauchers nach Art. 10 lit. b CISG auf dessen gewöhnlichen Aufenthalt an, für den Verordnungsvorschlag dagegen nach Art. 4(3)(a) DCESL-VO auf die angegebene Anschrift, die Rechnungsanschrift oder die Lieferanschrift des Verbrauchers, und dies könnte dazu führen, dass sich mit Blick auf Verbraucherverträge das UN-Kaufrecht und das Gemeinsame Europäische Kaufrecht in ihrer An- bzw. Verwendbarkeit nicht immer decken. Doch ist das UN-Kaufrecht, wie sofort deutlich werden wird, auf Verträge zwischen einem Unternehmer und einem Verbraucher ohnehin nur in Ausnahmefällen anwendbar.

\section{Verbraucher, Kleine und Mittlere Unternehmen und andere Unternehmer}

Nach Art. 1(3) CISG ist es für die Anwendbarkeit des UN-Kaufrechts ohne Bedeutung, ob die Parteien Kaufleute oder Nichtkaufleute sind oder ob der Vertrag handelsrechtlicher oder bürgerlich-rechtlicher Art ist. ${ }^{14}$ Die autoritative englische Fassung spricht etwa von der Unerheblichkeit des „civil or commercial character of the parties or of the contract". Doch werden Verträge zwischen Verbrauchern und Unternehmern in der Regel durch Art. 2(a) CISG vom Anwendungsbereich des UN-Kaufrechts ausgenommen, auch wenn die Begriffe des Unternehmers und des Verbrauchers in Art. 2(a) CISG nicht verwendet werden: ${ }^{15}$ Das UN-Kaufrecht „findet keine Anwendung auf den Kauf [...] von Ware für den persönlichen Gebrauch oder den Gebrauch in der Familie oder im Haushalt, es sei denn, dass der Verkäufer vor oder bei Vertragsabschluss weder wusste noch wissen musste, dass die Ware für einen solchen Gebrauch gekauft wurde“.

Das Gemeinsame Europäische Kaufrecht hat hier einen anderen Ansatz, der in Art. 7(1) DCESL-VO zum Ausdruck kommt:

„Das Gemeinsame Europäische Kaufrecht darf nur verwendet werden, wenn der Verkäufer [...] Unternehmer ist. Sind alle Parteien Unternehmer, kann das Gemeinsame Europäische Kaufrecht verwendet werden, wenn mindestens eine dieser Parteien ein kleines oder mittleres Unternehmen (,KMU') ist.“

Der Verkäufer muss also Unternehmer sein. Und auf Käuferseite darf grundsätzlich kein Unternehmer stehen. Das ergibt sich zwar schon jetzt aus dem zweiten Satz des Art. 7(1) DCESLVO. Und auch der Zusammenhang mit Art. 13(b) DCESL-VO (hierzu sogleich) legt dieses Verständnis nahe. Die Einfügung eines „nur“ im Wortlaut des Art. 7(1) CESL-VO wäre aber zu

9 Vgl. Eidenmüller/Jansen/Kieninger/Wagner/Zimmermann, JZ 2012, 274; Leible, in: Remien/Herrler/Limmer (o Fn. 3), S. 29.

10 So auch Schmidt-Kessel, in: ders. (o. Fn. 3), S. 34.

11 Siehe unten den Text zu und nach Fn. 42. Siehe außerdem meinen Beitrag im nächsten Heft der IHR unter Gliederungspunkt II.

12 Kritisch auch Eidenmüller/Jansen/Kieninger/Wagner/Zimmermann, JZ 2012, 274; Schmidt-Kessel (o. Fn. 10), S. 34. Als Vorteil verstehend Staudenmayer, NJW 2011, 3494. Differenzierend Leible (o. Fn. 9), S. 28 ff.

13 Vgl. Ferrari, in: Schlechtriem/Schwenzer, (o. Fn. 6), Art. 1 Rn. 46; Staudinger/Magnus (o. Fn. 7), Art.1 Rn. 62 ff.; Piltz, Internationales Kaufrecht, 2. Aufl. (2008), Rn. 2-77 ff. Aus der Rechtsprechung vgl. OLG Stuttgart, CISG-online Nr. 583.

14 Hierzu Schlechtriem, Internationales UN-Kaufrecht, 4. Aufl. 2007, Rn. 12.

15 Hierzu Ferrari, in: Schlechtriem/Schwenzer (o. Fn. 6), Art. 2 Rn. 7 ff.; Staudinger/Magnus (o. Fn. 7), Art. 2 Rn. 10 ff. 
erwägen: „Sind alle Parteien Unternehmer, kann das Gemeinsame Europäische Kaufrecht [nur] verwendet werden, [...].“

Damit ist das Gemeinsame Europäische Kaufrecht also bei Verträgen zwischen Unternehmern als Verkäufer und Verbrauchern als Käufer verwendbar. Auf solche Verträge ist das UNKaufrecht grundsätzlich nicht anwendbar. Da das UN-Kaufrecht in Art. 2(a) CISG mit der Wendung „Ware für den persönlichen Gebrauch oder den Gebrauch in der Familie oder im Haushalt" der Sache nach die Kriterien widerspiegelt, die auch für den Verbraucherbegriff des Art. 2(f) DCESL-VO bestimmend sind, wird es insoweit kaum zu Fällen kommen, in denen gleichzeitig das UN-Kaufrecht anwendbar und das Gemeinsame Europäische Kaufrecht verwendbar ist. Zu solchen Überlappungen kann es vor allem dann kommen, wenn der Unternehmer nicht wusste oder wissen musste, dass der Käufer die Ware etwa für seinen persönlichen Gebrauch kauft. ${ }^{16}$ Dann ist das UN-Kaufrecht auch auf Verbraucherverträge anwendbar. Für das Erkenntnisziel dieses Beitrages sind diese Fälle freilich ohne Bedeutung, werden die Schnittmengen zwischen UN-Kaufrecht und Gemeinsamem Europäischen Kaufrecht doch allein deshalb beleuchtet, um abschätzen zu können, in welchen Fällen sich die Parteien zwischen beiden Instrumenten bewusst entscheiden müssen. Ist das UNKaufrecht im Ergebnis aber nur auf Verbraucherverträge anwendbar, wenn der Verkäufer von der Verbrauchereigenschaft des Käufers keine Kenntnis hatte oder haben musste, wird er auch keine besondere Motivation entwickeln, in solchen Fällen das UN-Kaufrecht und nicht das Gemeinsame Europäische Kaufrecht zur Anwendung kommen zu lassen.

Im Fall, dass der Verkäufer Verbraucher oder zumindest kein Unternehmer, also etwa ein Idealverein, ist und der Käufer kein Verbraucher ist, ist das Gemeinsame Europäische Kaufrecht nicht verwendbar, das UN-Kaufrecht freilich anwendbar ${ }^{17}$ Auch in diesem Fall kommt es also zu keinen Überschneidungen im Geltungsbereich. Gleiches gilt für Verträge zwischen zwei Verbrauchern. Hier ist mangels Unternehmereigenschaft des Verkäufers das Gemeinsame Europäische Kaufrecht nach Art. 7(1) DCESL-VO nicht verwendbar und das UN-Kaufrecht nach Art. 2 (a) CISG in der Regel nicht anwendbar.

Überschneidungen ergeben sich dagegen zum einen in Fällen, in denen auf der einen Seite, gleich ob Verkäufer- oder Käuferseite, ein Unternehmer und auf der anderen Seite ein kleines oder mittleres Unternehmen (KMU) steht. Kleine und mittlere Unternehmen werden, vor allem wegen der mangelnden Erkennbarkeit für den Vertragspartner, auf nicht unproblematische Weise in Art. 7(2) DCESL-VO über ihre Mitarbeiterzahl und ihren Jahresumsatz bzw. ihre Jahresbilanzsumme definiert. ${ }^{18}$

Zum anderen ergeben sich Überschneidungen in Fällen, in denen auf Verkäuferseite ein Unternehmer und auf Käuferseite eine Person steht, die weder Verbraucher noch Unternehmer ist, also etwa ein Idealverein. Dass auch dieser Fall vom Gemeinsamen Europäischen Kaufrecht erfasst ist, bedarf einer kurzen Begründung. Denn solche Verträge werden weder in den Erwägungsgründen noch in der Begründung als Fallkonstellationen genannt, für die der Verordnungsentwurf ausgearbeitet worden ist. Ganz im Gegenteil sprechen die Begründung und die Erwägungsgründe als Regelungsgegenstand explizit nur von Verträgen zwischen Verbrauchern und Unternehmern einerseits und Verträgen unter Unternehmern andererseits. ${ }^{19}$ Verträge mit einem
Unternehmer auf Verkäuferseite und einer Person, die weder Verbraucher noch Unternehmer ist, auf Käuferseite werden auch im Kaufrechtsentwurf selbst konsequent ignoriert. ${ }^{20}$ Indes verlangt Art. 7(1) DCESL-VO nur, dass der Verkäufer ein Unternehmer ist. Nach dem allein maßgeblichen Art. 7(1) DCESL-VO sollte das Gemeinsame Europäische Kaufrecht also auch für Verträge zwischen einem Unternehmer als Verkäufer und etwa einem Idealverein als Käufer anwendbar sein. Dafür spricht weiterhin Art. 1 (2) DCESL-VO, der als Ziel des Verordnungsvorschlages bestimmt, dass die Verordnung „es Unternehmen [ermöglicht], sich bei allen ihren grenzübergreifenden Geschäften auf gemeinsame Vorschriften zu stützen und diesselben Vertragsbestimmungen zu verwenden“. Verträge mit Käufern, die weder Verbraucher noch Unternehmer sind, vom Geltungsbereich des Gemeinsamen Europäischen Kaufrechts auszuschließen, wäre mit diesem Ziel nicht vereinbar. Und die Ausklammerung solcher Verträge würde schließlich auch schlicht keinen Sinn machen. ${ }^{21}$

Steht auf beiden Seiten ein Unternehmer, ohne dass eine Partei ein kleineres oder mittleres Unternehmen ist, dann ist zwar das UN-Kaufrecht anwendbar, das Gemeinsame Europäische Kaufrecht indes zunächst nicht verwendbar. Doch soll den Mitgliedstaaten gemäß Art. 13(b) DCESL-VO die Möglichkeit eingeräumt werden, das Gemeinsame Europäische Kaufrecht auch auf solche Fälle zu erstrecken:

„Ein Mitgliedstaat kann beschließen, dass das Gemeinsame Europäische Kaufrecht für Verträge verwendet werden darf, [...] b) wenn alle Vertragsparteien Unternehmer sind, aber keiner davon ein KMU nach Maßgabe von Artikel 7 Absatz 2 ist."

Macht ein Mitgliedstaat von dieser Option Gebrauch, dann wären für Verträge zwischen Unternehmern sowohl das UN-Kauf-

16 Ausführlich Schroeter (o. Fn. 1), S. 143 ff. Vgl. auch Ferrari, in: Schlechtriem/Schwenzer (o. Fn. 6), Art. 2 Rn. 7 ff.; Staudinger/Magnus (o. Fn. 7), Art. 2 Rn. 29; Saenger, in: Bamberger/Roth, BGB, 2. Aufl. 2007, Art. 2 CISG Rn. 6; Spohnheimer, in: Kröll/Mistelis/Viscasillas, CISG, 2011, Art. 2 Rn. 21 ff. Freilich ist nicht ausgeschlossen, dass es in Randbereichen auch darüber hinaus zu unterschiedlichen Grenzziehungen kommt, so etwa in den Fällen eines sogenannten dual use. Für das UN-Kaufrecht geht die wohl h.M. dahin, dass von Art. 2(a) CISG nur Fälle erfasst sind, in denen die Ware allein dem persönlichen Gebrauch dienen soll: vgl. Ferrari, in: Schlechtriem/Schwenzer (o. Fn. 6), Art. 2 Rn. 12; Staudinger/Magnus (o. Fn. 7), Art. 2 Rn. 17; Piltz (o. Fn. 13), Rn. 2-61; jeweils mit Nachweisen auf die Gegenansicht. Fälle eines dual use werden daher vom UN-Kaufrecht erfasst. Für den europäischen und deutschen Verbraucherbegriff wird in der Regel auf den Schwerpunkt des Zwecks abgestellt: vgl. nur die Diskussion bei Micklitz, in: MünchKomm-BGB, 6. Aufl. 2012, \$13 Rn. 40 mit zahlreichen weiteren Nachweisen. Vgl. zu den Fällen des dual use auch Spohnheimer, a.a.O., Art. 2 Rn. 21 und ausführlich, freilich mit anderem Ergebnis, Schroeter, a.a.O.

17 Vgl. Staudinger/Magnus (o. Fn. 7), Art. 2 Rn. 18.

18 Eidenmüller/Jansen/Kieninger/Wagner/Zimmermann, JZ 2012, 270; Zimmermann, JBl 2012, 18; Pfeiffer, in: Remien/Herrler/Limmer (o. Fn. 3), S. 38; Ackermann, in: Remien/Herrler/Limmer (o. Fn. 3), S. 55; SchmidtKessel (o. Fn. 3), S. 8.

19 Siehe KOM (2011) 635 endg., S. 2 f., 13 der Begründung; Erwägungsgrund 21 DCESL-VO. Siehe außerdem Schulte-Nölke, in: Schulze/Stuyck (o. Fn. 4), S. 41.

20 Siehe unten den Text zu und nach Fn. 23. Siehe weiterhin meinen Beitrag im nächsten Heft der IHR unter Gliederungspunkten VII und VIII.

21 Siehe dazu meinen Beitrag im nächsten Heft der IHR unter Gliederungspunkt II. 
recht anwendbar als auch das Gemeinsame Europäische Kaufrecht verwendbar.

\section{Der Bezug zu den Vertragsstaaten des UN-Kaufrechts und zu den Mitgliedstaaten der Europäischen Union}

Wir können also für den persönlichen Geltungsbereich beider Instrumente folgende Schnittmengen feststellen: (1) Sind beide Parteien Unternehmer, so muss zumindest eine Partei ein kleines oder mittleres Unternehmen sein. (2) Ist nur der Verkäufer Unternehmer, so muss auf Käuferseite eine Person stehen, die weder Verbraucher noch Unternehmer ist, also etwa ein Idealverein. (3) Macht ein Mitgliedstaat von der Option des Art. 13(b) DCESL-VO Gebrauch, kann auf beiden Seiten ein Unternehmer stehen, ohne dass eine der Parteien ein kleines oder mittleres Unternehmen ist. Betrachten wir nur für diese Fälle nochmals die Regeln zur räumlichen An- bzw. Verwendbarkeit:

Nach Art. 1(1)(a) CISG müssen die Parteien ihre Niederlassungen zwar in verschiedenen Staaten haben, aber beide Staaten müssen Vertragsstaaten sein, damit das UN-Kaufrecht anwendbar ist. Die umstrittene und nach Art. 95 CISG auch nicht von allen Vertragsstaaten als verbindlich anerkannte Regel des Art. 1 (1)(b) CISG führt zudem dazu, dass das UN-Kaufrecht anwendbar ist, wenn das Kollisionsrecht auf das Recht eines Vertragsstaates verweist. ${ }^{22}$ Der Verordnungsvorschlag geht über Art. 1 (1)(a) CISG hinaus, als nach Art. 4(2) DCESL-VO ausreicht, wenn einer der Staaten, in denen eine der Vertragsparteien ihren gewöhnlichen Aufenthalt hat, ein Mitgliedstaat ist. Freilich ist Art. 4 (2) DCESL-VO nur einschlägig, wenn beide Vertragsparteien Unternehmer sind.

Eine Regelungslücke enthält Art. 4 DCESL-VO für den Fall, dass der Käufer weder Unternehmer noch Verbraucher ist. Art. 4 DCESL-VO regelt nur (1) Verträge zwischen zwei Unternehmern und (2) Verträge zwischen einem Unternehmer und einem Verbraucher. Nun sind die Begriffe Verbraucher und Unternehmer auch nach Art. 2(e) und (f) DCESL-VO nicht abschließend: ${ }^{23}$ Es gibt Personen, die weder Unternehmer noch Verbraucher sind, eben die bereits genannten Idealvereine. Sie handeln weder zum Zweck einer gewerblichen, geschäftlichen, handwerklichen oder beruflichen Tätigkeit, so dass sie keine Unternehmer i.S.d. Art. 2 (e) DCESL-VO sind. Soweit sie juristische Personen sind, unterfallen sie aber auch nicht dem Verbraucherbegriff des Art. 2(f) DCESL-VO, weil es sich dann bei ihnen nicht um eine natürliche Person handelt. Für Verträge zwischen einem Unternehmer als Verkäufer und einem Käufer, der weder Unternehmer noch Verbraucher ist, wird der Begriff des grenzübergreifenden Vertrages also nicht geregelt. Da juristische Personen in Art. 4(4) DCESLVO genannt sind, sollte diese Regelungslücke, sofern sie sich auch noch in der endgültigen Verordnung findet, über Art. 4(2) DCESL-VO geschlossen werden. Auch für solche Vertragsparteien sollte es folglich auf den gewöhnlichen Aufenthalt im Sinne des Art. 4(4)-(5) DCESL-VO ankommen.

Kommen beide Vertragsparteien aus solchen Mitgliedstaaten der Europäischen Union, die zugleich Vertragsstaaten des UNKaufrechts sind, so decken sich nach alledem der Geltungsbereich von UN-Kaufrecht und Gemeinsamen Europäischen Kaufrecht.
Darüber hinaus ist das Gemeinsame Europäische Kaufrecht auch in Fällen verwendbar, in denen die andere Vertragspartei aus einem Mitgliedstaat der Europäischen Union kommt, das nicht zugleich Vertragsstaat des UN-Kaufrechts ist, und zudem in solchen Fällen, in denen die andere Vertragspartei nicht einmal Mitgliedstaat der Europäischen Union ist. Und schließlich haben die Mitgliedstaaten nach Art. 13(a) DCESL-VO die Option, das Gemeinsame Europäische Kaufrecht auch für rein interne Sachverhalte zur Verfügung zu stellen. Wechseln wir von der Binnen- zur Außenperspektive, so läßt sich feststellen, dass für Marktteilnehmer, die ihren gewöhnlichen Aufenthalt nicht in einem Mitgliedstaat der Europäischen Union haben, das Gemeinsame Europäische Kaufrecht nur verwendbar ist, wenn ihr Vertragspartner seinen gewöhnlichen Aufenthalt in einem Mitgliedstaat hat.

\section{Warenkauf, Bereitstellung digitaler Inhalte und verbundene Dienstleistungen}

Das UN-Kaufrecht und das Gemeinsame Europäische Kaufrecht gelten für Warenkaufverträge: Art. 1(1) CISG und Artt. 3 und 5(a) DCESL-VO. Für das UN-Kaufrecht haben sich sowohl um den Warenbegriff als auch um den Begriff des Kaufvertrages eine reiche Rechtsprechung und zahlreiche Literaturmeinungen gebildet. ${ }^{24}$ Ob die Rechtsprechung für das Gemeinsame Europäische Kaufrecht immer die gleichen Wege gehen wird, bleibt abzuwarten. Zudem finden sich in beiden Regelwerken im Detail abweichende Bestimmungen: ${ }^{25}$ Für das UN-Kaufrecht ist bei dem Warenbegriff insbesondere die Einordnung von Software strittig. ${ }^{26}$ Nach Art. 5 (b) DCESL-VO sind Verträge über die Bereitstellung digitaler Inhalte vom Gemeinsamen Europäischen Kaufrecht erfasst, und zwar unabhängig davon wie und ob die Bereitstellung entgeltlich erfolgt. Dass die Art der Bereitstellung unerheblich ist, wird auch für das UN-Kaufrecht vertreten. ${ }^{27}$ Doch die unentgeltliche Bereitstellung wird nicht unter das UN-Kaufrecht fallen. Auch für Verträge, bei denen der Verkäufer neben der Lieferung von Waren noch andere Leistungen, etwa deren Herstellung schuldet, finden sich in Art. 3 CISG einerseits und Artt. 5(c), 6(1) DCESL-VO andererseits im Detail voneinander abweichende Regelungen. Für die Zwecke dieses Beitrages reicht freilich die Beobachtung aus, dass beide Regelwerke im Kern mit Warenkaufverträgen einen identischen Geltungsbereich haben. Auch die umstrittene Regel des Art. 6(2) DCESL-VO, wonach das Gemeinsame Europäische Kaufrecht nicht für Ratenkaufverträge verwendet werden darf,,28

22 Hierzu nur Ferrari, in: Schlechtriem/Schwenzer (o. Fn. 6), Art. 1 Rn. 69 ff.

23 Zur Verbraucherdefinition kritisch Weller, in: Schmidt-Kessel (o. Fn. 3), S. $149 \mathrm{f}$.

24 Vgl. etwa Ferrari, in: Schlechtriem/Schwenzer (o. Fn. 6), Art. 1 Rn. 12 ff.; Staudinger/Magnus (o. Fn. 7), Art. 1 Rn. 12 ff.; Schlechtriem (o. Fn. 14), Rn. 24 ff.; Piltz (o. Fn. 13), Rn. 2-19 ff.; jeweils mit Nachweisen aus der Rechtsprechung.

25 Vgl. zum Ganzen auch Pfeiffer (o. Fn. 18), S. 40 ff.

26 Vgl. nur Ferrari, in: Schlechtriem/Schwenzer (o. Fn. 6), Art. 1 Rn. 38.

27 Vgl. Ferrari, in: Schlechtriem/Schwenzer (o. Fn. 6), Art. 1 Rn. 38 Fn. 165; Staudinger/Magnus (o. Fn. 7), Art. 1 Rn. 4; Piltz (o. Fn. 13), Rn. 2-48; Saenger, in: Bamberger/Roth (o. Fn. 16), Art. 1 Rn. 7. Offenlassend Mistelis, in: Kröll/Mistelis/Viscasillas (o. Fn. 16), Art. 1 Rn. 40.

28 Kritisch etwa Eidenmüller/Jansen/Kieninger/Wagner/Zimmermann, JZ 2012, 275; Schmidt-Kessel (o. Fn. 10), S. 32. Erläuternd Staudenmayer, NJW 2011, 3494. 
ist für den vorliegenden Beitrag ohne Bedeutung, gilt Art. 6(2) DCESL-VO doch nur für Verbraucherverträge.

\section{Ausschluss des UN-Kaufrechts und Verwendbarkeit des Gemeinsamen Europäischen Kaufrechts}

Das UN-Kaufrecht ist ohne Weiteres anwendbar, soweit dessen Anwendungsbereich eröffnet ist. Die Vertragsparteien können dessen Geltung indes nach Art. 6 CISG ausschließen. ${ }^{29}$ Der Verordnungsvorschlag spricht nicht von der Anwendbarkeit des Gemeinsamen Europäischen Kaufrechts, sondern von dessen Verwendbarkeit. Wollen es die Parteien zur Anwendung kommen lassen, müssen sie dessen Geltung nach Artt. 3 und 8(1) DCESL-VO vereinbaren. ${ }^{30}$ An diese Vereinbarung stellen Artt. 8 (2)-(3) und 9 DCESL-VO besondere Anforderungen, ${ }^{31}$ die allerdings nur zu beachten sind, wenn das Gemeinsame Europäische Kaufrecht zwischen einem Verbraucher und einem Unternehmer verwendet werden soll. Diese Fälle interessieren hier nicht. Im Übrigen gilt, dass auch eine konkludente Vereinbarung über die Verwendung des Gemeinsamen Europäischen Kaufrechts möglich ist ${ }^{32}$ und dass eine Verwendungsvereinbarung zugleich ein konkludenter Ausschluss der Anwendung des UN-Kaufrechts sein soll. ${ }^{33}$

\section{Die Vorschaltlösung des Gemeinsamen Europäischen Kaufrechts}

Das UN-Kaufrecht bestimmt seine Anwendbarkeit in Art.1(1)(a) CISG autonom, das heißt ohne dass es darauf ankommt, dass das Internationale Privatrecht auf das Recht eines Vertragsstaates des UN-Kaufrechts verweist. ${ }^{34}$ Daneben kann das UN-Kaufrecht nach Art. 1(1)(b) CISG auch kraft kollisionsrechtlicher Verweisung auf das Recht eines Vertragsstaats anwendbar sein, die sogenannte Vorschaltlösung. ${ }^{35}$

Der Verordnungsvorschlag wählt dagegen allein die Vorschaltlösung und verfolgt damit folgendes Ziel: ${ }^{36}$ Das Gemeinsame Europäische Kaufrecht solle nicht als sogenanntes 28. Kaufrechtssystem neben die Rechte der Mitgliedstaaten treten. Vielmehr sei entscheidend, dass das Gemeinsame Europäische Kaufrecht als Verordnungsrecht in jedem Mitgliedstaat unmittelbar zur Anwendung komme und damit in jedem Mitgliedstaat als zweites Kaufrecht neben das bereits bestehende mitgliedstaatliche Kaufrecht trete. Als Folge hänge die Verwendbarkeit des Gemeinsamen Europäischen Kaufrechts davon ab, dass das Recht eines Mitgliedstaates kollisionsrechtlich anwendbar sei. Nur wenn nach den Regeln des Internationalen Privatrechts das Recht eines Mitgliedstaates anwendbar sei, stehe den Parteien überhaupt die Wahl zwischen dem mitgliedstaatlichen Kaufrecht und dem Gemeinsamen Europäischen Kaufrecht offen.

Die Prüfung, ob das Gemeinsame Europäische Kaufrecht verwendbar ist, muss also immer in drei Schritten erfolgen. Zunächst ist zu prüfen, ob das Recht eines Mitgliedstaates anwendbar ist. Sodann ist zu prüfen, ob das Gemeinsame Europäische Kaufrecht nach Artt. 4-7 DCESL-VO verwendbar ist. Und schließlich müssen die Parteien sich auf die Verwendung des
Gemeinsamen Europäischen Kaufrechts nach Artt. 3, 8-9 DCESL-VO geeinigt haben. Dem im ersten Schritt ermittelten anwendbaren mitgliedstaatlichen Recht kommt damit eine doppelte Funktion zu: Es ist der Geltungsrahmen des Gemeinsamen Europäischen Kaufrechts. Und auf das mitgliedstaatliche Recht muss zurückgegriffen werden, um externe Lücken des Gemeinsamen Europäischen Kaufrechts zu schließen. ${ }^{37}$

Diese Vorschaltlösung, die im Verordnungsvorschlag nur in den Erwägungsgründen, aber nicht im eigentlichen Regeltext verankert ist, ${ }^{38}$ ist zu Recht aus unterschiedlichen Gründen kritisiert worden. ${ }^{39}$ Sie erklärt sich vor allem mit kompetenzrechtlichen Erwägungen. Zudem will die Kommission so Probleme um die Anwendung des Art. 6(2) Rom I-VO umgehen. Die Vorschaltlösung stellt zudem einen Anreiz für die Mitgliedstaaten dar: nach Art. 13(b) DCESL-VO können die Mitgliedstaaten das Gemeinsame Europäische Kaufrecht auch für den Unternehmerverkehr öffnen. ${ }^{40}$ Nur ein einziger Mitgliedstaat muss von dieser Option Gebrauch machen. ${ }^{41}$ Wählen die Vertragsparteien sodann das Recht dieses Mitgliedstaates, ist das Gemeinsame Europäische Kaufrecht selbst dann verwendbar, wenn beide Unternehmer aus Mitgliedstaaten kommen, die von der Möglichkeit des Art. 13(b) DCESL-VO keinen Gebrauch gemacht haben. Sollte sich andeuten, dass das Gemeinsame Europäische Kaufrecht für den Unternehmerverkehr attraktiv ist, werden Mitgliedstaaten die Option des Art. 13(b) DCESL-VO nutzen. Denn anderenfalls liefen sie Gefahr, dass ihr Recht zwischen Unternehmern abgewählt und das eines Mitgliedstaates, das diese Möglichkeit genutzt hat, gewählt wird. Die Mitgliedstaaten könnten dadurch eine Schwächung ihres Rechtsstandortes fürchten. Denn das eigene Recht wäre zumindest noch bei externen Regelungslücken anzuwenden.

29 Siehe nur Ferrari, in: Schlechtriem/Schwenzer (o. Fn. 6), Art. 6 Rn. 5.

30 Hierzu kritisch Lando (o. Fn. 5), S. 19: „My fear ist that as an opt-in model the CESL will suffer the same fate [that of the PICC, the PECL and the ULIS/ULFIS in the UK]. "Vgl. außerdem Mankowski, IHR 2012, 1-2.

31 Hierzu kritisch Eidenmüller/Jansen/Kieninger/Wagner/Zimmermann, JZ 2012, 276; Zimmermann, JBl 2012, 19; Leible (o. Fn. 9), S. 31; Schmidt-Kessel (o. Fn.10), S. 41 ff.; Riesenhuber, GPR 2012, 2 ff.; Fornasier, RabelsZ 76 (2012), 433 ff. Erläuternd Staudenmayer, NJW 2011, 3494.

32 Hierzu kritisch Eidenmüller/Jansen/Kieninger/Wagner/Zimmermann, JZ 2012, 276.

33 Vgl. Staudenmayer, NJW 2011, 3495; Moser, in: Remien/Herrler/Limmer (o. Fn. 3), S. 13; Lando (o. Fn. 5), S. 15 f. Einschränkend Hesselink, ERPL 2012, 201 f. Zu möglichen Anwendungskonflikten vgl. Leible (o. Fn. 9), S. 32 f.; Schmidt-Kessel (o. Fn. 3), S. 22 f.

34 Siehe nur Ferrari, in: Schlechtriem/Schwenzer (o. Fn. 6), Art. 1 Rn. 63 ff.

35 Siehe nur Staudinger/Magnus (o. Fn. 7), Art. 1 Rn. 93 ff.

36 Vgl. zum folgenden Fornasier, RabelsZ 76 (2012), 401 ff. Leible (o. Fn. 9), S. 22 ff.; Zimmermann, JBl 2012, 9; Staudenmayer, NJW 2011, 3494 f.; Schmidt-Kessel (o. Fn. 10), S. 37 ff.; Moser (o. Fn. 33), 13; Hesselink, ERPL 2012, 195 ff. Vgl. außerdem Heiss, in: Festschr. F. Günter H. Roth, 2011, S. 237-246.

37 Siehe dazu meinen Beitrag im nächsten Heft der IHR unter Gliederungspunkt III.

38 Vgl. Erwägungsgründe 9-10 DCESL-VO. Hierzu kritisch Fornasier, RabelsZ 76 (2012), $419 \mathrm{f}$.

39 So von Tamm, VuR 2012, 4; Mankowski, RIW 2012, 100; Eidenmüller/ Jansen/Kieninger/Wagner/Zimmermann, JZ 2012, 273-276; Remien (o. Fn. 3), S. 3; Fornasier, RabelsZ 76 (2012), 420 ff. Positiv bewertend Leible (o. Fn. 9), S. $23 \mathrm{ff}$.

40 Siehe oben den Absatz nach Fn. 21

41 Staudenmayer, NJW 2011, 3494; Eidenmüller/Jansen/Kieninger/Wagner/Zimmermann, JZ 2012, 274; Pfeiffer (o. Fn. 18), S. 42. 
Die Vorschaltlösung setzt schließlich ein Fragezeichen hinter einen anderen Aspekt der Vorschriften des Verordnungsvorschlags über die Verwendbarkeit des Gemeinsamen Europäischen Kaufrechts: ${ }^{42}$ Warum ist es nach Art. 4(2) und (3) DCESL-VO notwendig, dass eine der Vertragspartei ihren gewöhnlichen Aufenthalt oder eine ihrer Anschriften in einem Mitgliedstaat der Europäischen Union hat? Wenn das Gemeinsame Europäische Kaufrecht im jeweiligen Mitgliedstaat als zweites Kaufrechtsregime gilt, so sollte es doch auch dann verwendet werden können, wenn keine der Vertragsparteien ihren gewöhnlichen Aufenthalt in einem Mitgliedstaat hat, aber wenn sie das Recht eines Mitgliedstaates wählen. Weil der Verordnungsvorschlag von einer Vorschaltlösung ausgeht und weil das Gemeinsame Europäische Kaufrecht nur zu Geltung kommt, wenn es gewählt wird, sollten die gegen Art. 1(1)(b) CISG vorgebrachten Bedenken nicht greifen. ${ }^{43}$ Zudem kombiniert der Verordnungsvorschlag der Sache nach die aus Art. 1(1)(a) und (b) CISG bekannten Anwendungsvoraussetzungen. Beide Alternativen sollen eine Verknüpfung eines Vertrages zu einem Vertragsstaat herstellen. ${ }^{44}$ Nach Art. 1(1)(a) müssen beide Parteien entweder ihre Niederlassungen in Vertragsstaaten haben, oder nach Art. 1 (1)(b) CISG muss das Recht eines Vertragsstaates kollisionsrechtlich anwendbar sein. Der Verordnungsvorschlag verlangt dagegen eine doppelte Verbindung des Vertrages mit Mitgliedstaaten: Das Recht eines Mitgliedstaates muss anwendbar sein, und zusätzlich muss eine Partei ihren gewöhnlichen Aufenthalt in einem Mitgliedstaat haben. Warum eine solche doppelte Verbindung notwendig sein soll, erschließt sich mir nicht. ${ }^{45}$

\section{Zusammenfassung}

Sowohl das UN-Kaufrecht als auch das geplante Gemeinsame Europäische Kaufrecht stellen Regeln für grenzüberschreitende Kaufverträge bereit. Nach Inkrafttreten des Gemeinsamen Europäischen Kaufrechts wird sich den Marktteilnehmern daher die Frage stellen, ob sie dieses oder das UN-Kaufrecht anwenden wollen. Freilich haben die Marktteilnehmer diese Wahl nur in den Fällen, in denen auch beide Instrumente an- bzw. verwendbar sind. Ziel dieses Beitrages war daher, die Schnittmengen beider Instrumente herauszuarbeiten. Für den persönlichen Geltungsbereich beider Regelwerke konnten wir folgende Überschneidungen feststellen: (1) Sind beide Parteien Unternehmer, so muss zumindest eine Partei ein kleines oder mittleres Unternehmen sein. (2) Ist nur der Verkäufer Unternehmer, so muss auf Käuferseite eine Person stehen, die weder Verbraucher noch Unternehmer ist, also etwa ein Idealverein. (3) Macht ein Mitgliedstaat von der Option des Art. 13(b) DCESL-VO Gebrauch, kann auf beiden Seiten ein Unternehmer stehen, ohne dass eine der Parteien ein kleines oder mittleres Unternehmen ist. Für all diese Verträge knüpft das UN-Kaufrecht an die Niederlassung der Parteien an. Für den Verordnungsvorschlag ist dagegen der gewöhnliche Aufenthalt maßgeblich. Doch führt dies nicht dazu, dass die Geltungsbereiche beider Instrumente sich nicht decken, stimmen doch die Konkretisierungen des gewöhnlichen Aufenthalts in Art. 4(4)-(5) DCESL-VO mit der Auslegung des Niederlassungsbegriffs der Artt. 1 und 10 CISG weitgehend überein. Betrachtet man den sachlichen Geltungsbereich so regeln beide Instrumente im Kern Warenkaufverträge. Nur in Randbereichen können die Geltungsbereiche auseinanderfallen, weil der durch Rechtsprechung und Literatur entwickelte Warenbegriff des UNKaufrechts nicht zwingend für das Gemeinsame Europäische Kaufrecht passt und weil beide Instrumente in den Randbereichen unterschiedliche Vertragstypen miterfassen. Unterschiede bestehen zwischen beiden Instrumenten mit Blick auf den Bezug, der zu den Vertragsstaaten des UN-Kaufrechts bzw. zu einem Mitgliedstaat der Europäischen Union bestehen muss.

\footnotetext{
42 Kritisch auch Fornasier, RabelsZ 76 (2012), 422.

43 Vgl. die Darstellung bei Staudinger/Magnus (o. Fn. 7), Art. 1 Rn. 94 f.

44 Vgl. hierzu nur Staudinger/Magnus (o. Fn. 7), Art. 1 Rn. 3, 83 f.; Ferrari, in: Schlechtriem/Schwenzer (o. Fn. 6), Art. 1 Rn. 61 f.

$45 \mathrm{Zu}$ den praktischen Konsequenzen siehe meinen Beitrag im nächsten Heft der IHR unter Gliederungspunkt II.
}

\section{Entscheidungen}

\section{UN-Kaufrecht (CISG)}

\section{Art. 35, 45, 74 CISG}

1. Einer zwischen den Parteien abgesprochenen Schriftformabrede kommt im Zweifel konstitutive Bedeutung zu. 2. Vom Handelsbrauch abweichende Zusagen zur Qualität der zu liefernden Ware müssen unzweifelhaft und ausdrücklich erklärt werden.
3. Zur Ermittlung des gewöhnlichen Gebrauchszweckes der zu liefernden Ware ist auf die im Land des Verkäufers herrschenden Standards abzustellen.

[Leitsätze der Redaktion]

Deutschland: OLG Hamm, Urteil vom 30.11.2010, I-19 U 147/09

(Vorinstanz: LG Münster, Urteil vom 15.10.2009 4 O 200/07) 